Marquette University

e-Publications@Marquette

Chemistry Faculty Research and Publications

Chemistry, Department of

$1-16-1998$

\title{
Studies on the Preparation of Protomycinolide IV: Enantioselective Synthesis of the C3-C9 Segment
}

James Thomas Wasicak

Marquette University

William Donaldson

Marquette University, william.donaldson@marquette.edu

Accepted version. Tetrahedron: Asymmetry, Vol.9, No. 1 (January 16. 1998): 133-140. DOI. (C) 1998 Elsevier Science Ltd. Used with permission. 
Marquette University

e-Publications@Marquette

\section{Chemistry Faculty Research and Publications/College of Arts and Sciences}

This paper is NOT THE PUBLISHED VERSION; but the author's final, peer-reviewed manuscript. The published version may be accessed by following the link in th citation below.

Tetrahedron : Asymmetry, Vol. 9, No. 1 (January 16, 1998): 133-140. DOI. This article is (C) Elsevier and permission has been granted for this version to appear in e-Publications@Marquette. Elsevier does not grant permission for this article to be further copied/distributed or hosted elsewhere without the express permission from Elsevier.

\section{Studies on the Preparation of Protomycinolide IV: Enantioselective Synthesis of the C3-C9 Segment}

James T. Wasicak

Department of Chemistry, Marquette University, Milwaukee, WI

William A. Donaldson

Department of Chemistry, Marquette University, Milwaukee, WI

\section{Abstract}

The C3-C9 segment, (-)-16, of the polyene macrolide antibiotic protomycinolide IV (1a) was prepared in optically pure form from commercially available methyl (S)-2-methyl-3-hydroxypropionate in 12 steps giving $17 \%$ yield.

\section{Introduction}

Protomycinolide IV and mycinolide IV (1a and $\mathbf{1 b}$ ) are 16-membered polyene macrolides isolated from the culture broth of Micromonospora griseorubida sp. nov. [1 $]^{\mathrm{a}}$ The configuration of $\mathbf{1} \mathbf{b}$ was determined by X-ray diffraction analysis $[1]^{b}$ Conversion of $\mathbf{1 b}$ into $\mathbf{1 a}$ (tosylation of the $1^{\circ}$ alcohol followed by reduction) 
demonstrated their structural association. While protomycinolide does not possess notable antibacterial activity, it has been shown to be a biosynthetic intermediate for the mycinamycin family of antibiotics which display significant activity against Gram-positive bacteria.[1] ${ }^{c}$ Structural correlation between protomycinolide IV and other 16-membered macrolides (e.g. chalcomycin, $[2]^{a}$ rosaramicin[2] $]^{b}$ and tylosin[2] ) has recently led to a proposed biosynthetic pathway for their formation. [2] ${ }^{a}$ In support of this mechanism, methyl mycinoate II (2a) and dicarboxymycinonic acid III (2b) have been isolated from the culture broth of a mutant of Micromonospora griseorubida which is unable to produce $\mathbf{1} \mathbf{a} .\left[^{[1]^{d}}\right.$ The total syntheses of $\mathbf{1 a},[3] \mathbf{2 a}$ and $\mathbf{b}[4]$ have been reported.

We recently described a synthetic strategy for the preparation of dienolide 1 which relies on the ability of an $\mathrm{Fe}(\mathrm{CO})_{3}$ moiety to control $\mathrm{C}-\mathrm{C}$ bond formation adjacent and remote to a coordinated diene (Scheme. 1). $[5]$ To this end, we have reported the preparation of the C10-C17 segment 3 from (methyl 3,5-hexadienoate)Fe(CO) ${ }_{3}$ in a highly diastereoselective fashion, as well as model studies for generation of the C9-C10 dienone bond via Friedel-Crafts acylation. The synthesis of 1 a by this methodology requires the functionalized acyl halide 4 . We herein report the preparation of the C3-C9 segment in an enantioselective fashion.
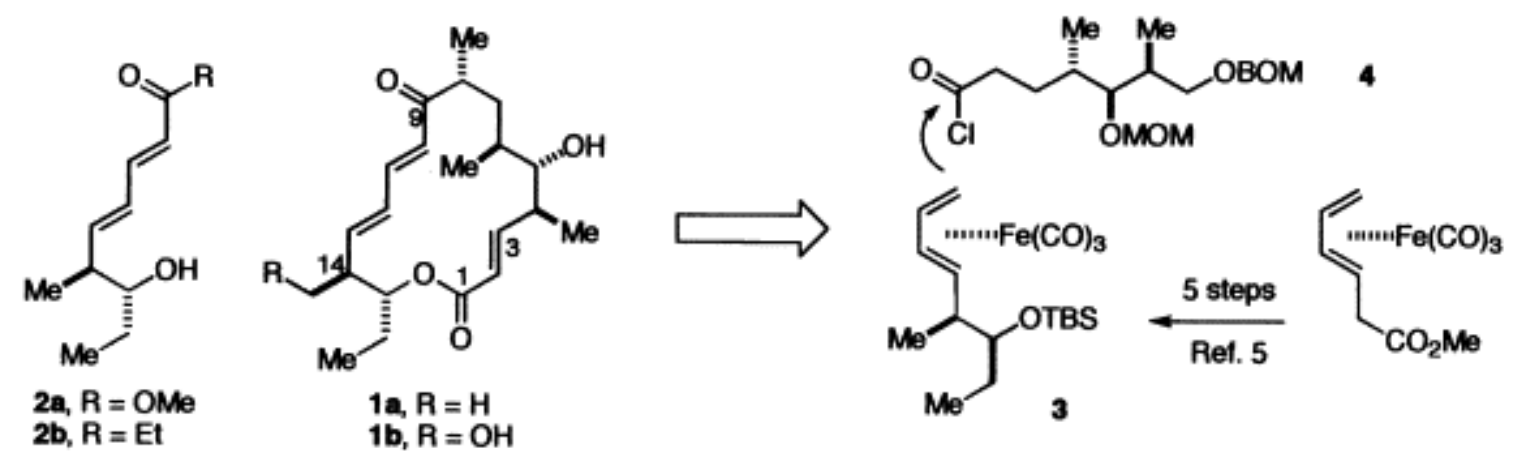

Scheme. 1.

\subsection{Enantioselective preparation of the C3-C9 segment}

The preparation of $\mathbf{1 7}$, in optically pure form, initially required preparation of the $\mathrm{C} 4-\mathrm{C} 6$ segment. There are many possibilities for generation of this stereotriad[6] and a double diastereoselective aldol condensation was chosen as our point of inception. Aldehyde (+)-7 was prepared from commercially available methyl (S)-2-methyl3-hydroxypropionate by protection, reduction[7] and oxidation[7] (70\% yield, 3 steps, Scheme. 2). Aldol condensation of (+)-7 with $N$-propionyl-4(R)-methyl-5(S)-phenyloxazolidin-2-one[8] was effected by the use of dibutylboron triflate/NEt ${ }_{2} \mathrm{PPr}$. The proton NMR spectrum of the crude product revealed one major diastereomer and a trace amount of at least one other diastereomer; however, the quantity of this very minor diastereomer was insufficient to determine its relative configuration or to determine a diastereomeric ratio. Chromatographic purification gave pure (+)-8 as a crystalline product (89\%). The 'anti-Felkin-syn-aldol' stereochemistry, which was expected on the basis of literature precedents, [9] was confirmed by X-ray diffraction analysis (Fig. 1).[10] 


$$
\text { RoO } \underbrace{M e}_{R}
$$

a $4, \mathrm{R}^{\prime}=\mathrm{H}_{1} \mathrm{R}=\mathrm{CO}_{2} \mathrm{Me}$

$(+)-5, \mathrm{R}^{\prime}=$ TBDPS, $\mathrm{R}=\mathrm{CO}_{2} \mathrm{Me}$

$(+)-6, \mathrm{R}^{\prime}=$ TBDPS, $\mathrm{R}=\mathrm{CH}_{2} \mathrm{OH}$

c $\zeta(+)-7, R^{\prime}=$ TBDPS, $R=C H O$

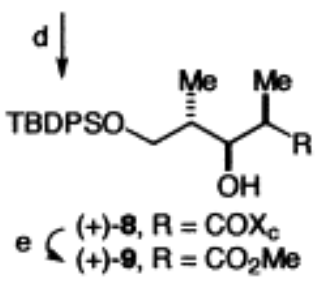

Scheme. 2. Reagents: (a) TBDPSCl/imidazole (93\%); (b) DIBAL/THF (83\%); (c) DMSO/(COCl) $/ \mathrm{NEt}_{3}(88 \%)$; (d) (N)propyl-4(R)-methyl-5(S)-phenyloxazolidinone/nBu $2 \mathrm{BOTf}$ (89\%); (e) $\mathrm{NaOMe} / \mathrm{MeOH}$ (78\%)

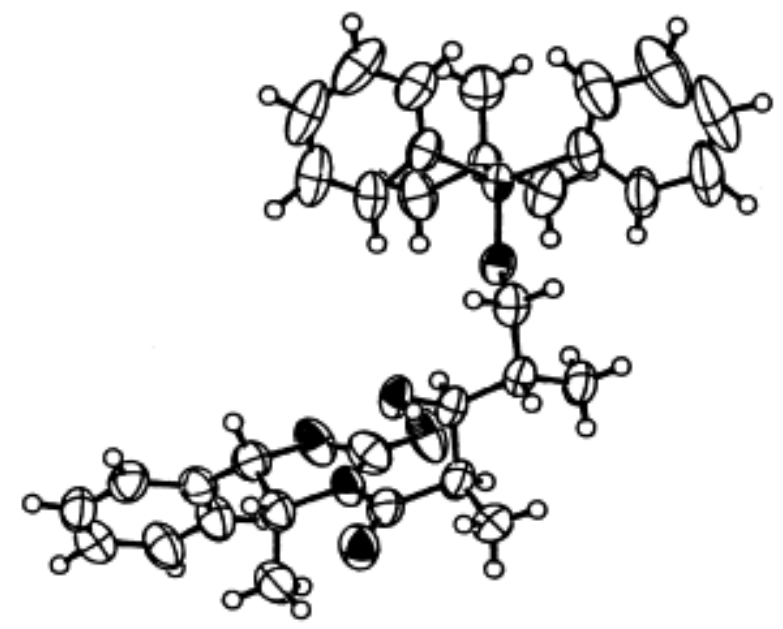

Fig. 1. ORTEP view of $(+)-8$

Treatment of (+)-8 with $\mathrm{NaOMe} / \mathrm{MeOH}$ gave the methyl ester (+)-9 (78\%) along with the recovered chiral auxilary $(75 \%$, Scheme. 2). Protection of the secondary alcohol with methoxymethyl chloride gave the ether (+)10 (95\%, Scheme. 3). Reduction of ester (+)-10 with DIBAL (THF/toluene) gave the alcohol (+)-11 (92\%), which was protected as its benzyloxymethyl ether (+)-12 (69\%). Removal of the silyl protecting group with TBAF (83\%) followed by Swern oxidation (84\%) gave the aldehyde (-)-14. Horner-Emmons olefination of (-)-14 with triethyl phosphonoacetate gave the unsaturated ester 15(88\%) which contains all of the carbons of the C3-C9 segment of 1. Brief ( $1 \mathrm{~h})$ catalytic hydrogenation of $\mathbf{1 5}$ resulted in saturation of the $\mathrm{C} 6-\mathrm{C} 7$ olefin without removal of the BOM protecting group (79\%). Hydrolysis of (-)-16 gave the carboxylic acid $\mathbf{1 7}(51 \%)$. The use of this segment in the synthesis of $\mathbf{1}$ will be reported in due course.

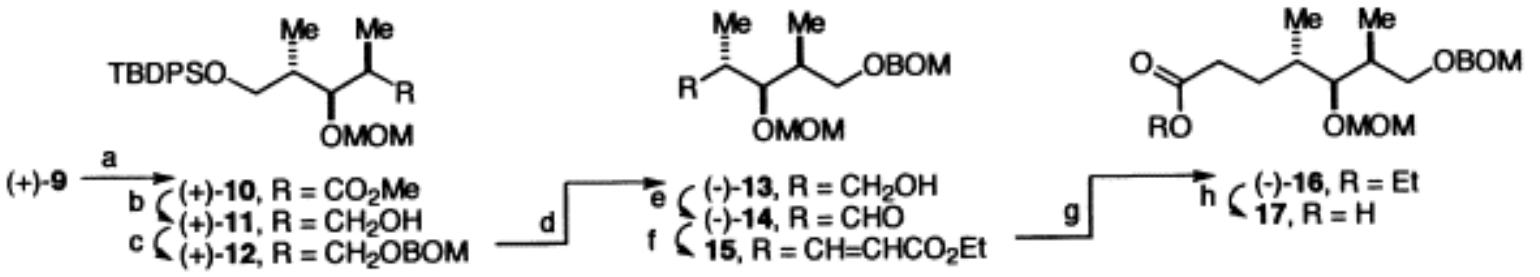

Scheme. 3. Reagents: (a) MOMCl/iPr 2 NEt (95\%); (b) DIBAL (92\%); (c) BOMCl/iPr 2 NH (69\%); (d) TBAF/THF (83\%); (e) DMSO/(COCl) $2 / \mathrm{NEt}_{3}$ (84\%); (f) (EtO) ${ }_{2} \mathrm{P}(\mathrm{O}) \mathrm{CH}_{2} \mathrm{CO}_{2} \mathrm{Et} / \mathrm{NaH}$ (88\%); (g) $\mathrm{H}_{2} / 10 \% \mathrm{Pd}-\mathrm{C}$ (79\%); (h) KOH/ $\mathrm{H}_{2} \mathrm{O} / \mathrm{EtOH}$ (51\%) 


\section{Experimental section}

\subsection{General data}

Spectrograde solvents were used without purification with the exception of tetrahydrofuran which was distilled from the sodium benzophenone ketyl. Aldrich sure-seal dichloromethane and DMSO were used without further purification. Methyl (S)-3-hydroxy-2-methylpropionate and 4(R)-methyl-5(S)-phenyloxazolidin-2-one were purchased from the Aldrich Chemical Company, Inc. Column chromatography was performed on silica gel 60 (0.04-0.063 mm, E. Merck or 60-200 mesh, Aldrich) and 'flash' chromatography was performed on silica gel 60 (230-400 mesh). Thin layer chromatography was performed on Merck (Kieselgel 60 F254) or Kodak Chromagram (silica gel without fluorescent indicator).

All ${ }^{1} \mathrm{H}$ NMR and ${ }^{13} \mathrm{C}$ NMR spectra were recorded at 300 and $75 \mathrm{MHz}$ respectively. Melting points were obtained on a Mel-Temp melting point apparatus and are uncorrected. Elemental analyses were obtained from Robertson Microlit Laboratories, Inc., Madison, NJ and high resolution mass spectra (EI) were obtained from the Washington University Resource for Mass Spectrometry.

\subsection{Methyl (S)-3-(tert-butyldiphenylsilyloxy)-2-methylpropionate ((+)-5)}

This was prepared from (S)-3-hydroxy-2-methylpropionate in a fashion similar to the procedure of Ley et al.[7] (93\%) and was identified by comparison to the literature ${ }^{1} \mathrm{H}$ NMR spectral data: $[\alpha]_{D}+17$ (c $2.5, \mathrm{CHCl}_{3}$ ), (lit.[7] $[\alpha]_{D}+23\left(c 7.8, \mathrm{CH}_{3} \mathrm{OH}\right)$ ); $\mathrm{R}_{f} 0.38$ (hexane:ethyl acetate=15:1).

\section{3. (R)-3-(tert-Butyldiphenylsilyloxy)-2-methyl-1-propanol ((+)-6)}

This was prepared by the reduction of (+)-5 with DIBAL in a fashion similar to the procedure of Ley et al.[7] (83\%) and was identified by comparison to the literature ${ }^{1} \mathrm{H}$ NMR spectral data: $[\alpha]_{\mathrm{D}}+6.1$ (c $\left.2.5, \mathrm{CHCl}_{3}\right)$, $\left(\right.$ lit. $[7][\alpha]_{\mathrm{D}}+6.3$ (c 1.0, $\left.\mathrm{CHCl}_{3}\right)$ ); $\mathrm{R}_{f} 0.27\left(\mathrm{CH}_{2} \mathrm{Cl}_{2}\right)$.

\section{4. (S)-3-(tert-Butyldiphenylsilyloxy)-2-methylpropanal ((+)-7)}

This was prepared by oxidation of (+)-6 with $\mathrm{DMSO} /(\mathrm{COCl})_{2}$ in a fashion similar to the procedure of Roush et al.[7] The crude product was passed through a 3.5 inch bed of $\mathrm{SiO}_{2}\left(\mathrm{CH}_{2} \mathrm{Cl}_{2}\right)$ to give (+)-7 as a waxy solid (88\%) which was identified by comparison to the literature ${ }^{1} \mathrm{H}$ NMR spectral data: $\mathrm{mp} 61-63^{\circ} \mathrm{C}$ (lit. [7] $]^{\mathrm{a}} \mathrm{mp} 51-61^{\circ} \mathrm{C}$ ); $[\alpha]_{D}+22$ (c 1.0, $\mathrm{CHCl}_{3}$ ); $\mathrm{R}_{f} 0.20$ (hexanes: $\mathrm{CH}_{2} \mathrm{Cl}_{2}=2: 1$ ).

\subsection{Chiral aldol product $((+)-8)$}

To a solution of $\mathrm{N}$-propionyl-4(R)-methyl-5(S)-phenyloxazolidin-2-one (36.6 g, $157 \mathrm{mmol}$ ) in $\mathrm{CH}_{2} \mathrm{Cl}_{2}(315 \mathrm{~mL})$ cooled to $0^{\circ} \mathrm{C}$ was added dropwise freshly distilled dibutylboron triflate $(47.3 \mathrm{~g}, 173 \mathrm{mmol})$ followed immediately by diisopropylethylamine $(32.8 \mathrm{~mL}, 188 \mathrm{mmol})$. The mixture was stirred for $30 \mathrm{~min}$, then cooled to $-78^{\circ} \mathrm{C}$ whereupon a solution of $(+)-7$ in $\mathrm{CH}_{2} \mathrm{Cl}_{2}(157 \mathrm{~mL})$ was added to the reaction mixture via a cannula transfer. The mixture was stirred for $30 \mathrm{~min}$, warmed to $\mathrm{rt}$, and stirred for an additional $90 \mathrm{~min}$. The mixture was cooled to $0^{\circ} \mathrm{C}$ and $\mathrm{pH} 7$ buffer $(225 \mathrm{~mL})$, methanol $(600 \mathrm{~mL})$ and $30 \% \mathrm{H}_{2} \mathrm{O}_{2}$ :methanol $(1: 1,225 \mathrm{~mL})$ were added in succession. The mixture was warmed to $\mathrm{rt}$ and stirred for $30 \mathrm{~min}$, after which the phases separated. The aqueous layer was extracted twice with $\mathrm{CH}_{2} \mathrm{Cl}_{2}$, and the combined organic phases were dried $\left(\mathrm{MgSO}_{4}\right)$ and concentrated. The residue was purified by flash chromatography (hexanes:ethyl acetate=12:1 to 8:1 gradient) to give (+)-8 as a colorless solid (78.4 g, 89\%): $\mathrm{mp} 102-103^{\circ} \mathrm{C} ;[\alpha]_{\mathrm{D}}+22$ (c 1.2, $\mathrm{CHCl}_{3}$ ); $\mathrm{R}_{f} 0.18$ (hexanes:ethyl acetate=6:1); ${ }^{1} \mathrm{H}$ NMR $\left(\mathrm{CDCl}_{3} / \mathrm{D}_{2} \mathrm{O}\right)$ 8 7.70-7.66 (m, 4H), 7.44-7.37 (m, 9H), 7.33-7.30 (m, 2H), $5.68(\mathrm{~d}, J=7.0 \mathrm{~Hz}$, $1 \mathrm{H}), 4.78(\mathrm{dq}, 7.0,6.6 \mathrm{~Hz}, 1 \mathrm{H}), 4.00-3.93(\mathrm{~m}, 2 \mathrm{H}), 3.82(\mathrm{dd}, J=4.4,9.9 \mathrm{~Hz}, 1 \mathrm{H}), 3.73(\mathrm{~d}, J=6.6,9.9 \mathrm{~Hz}, 1 \mathrm{H}), 1.90-$ $1.80(\mathrm{~m}, 1 \mathrm{H}), 1.25(\mathrm{~d}, \mathrm{~J}=6.6 \mathrm{~Hz}, 3 \mathrm{H}), 1.06(\mathrm{~s}, 9 \mathrm{H}), 0.93(\mathrm{~d}, J=7.0 \mathrm{~Hz}, 3 \mathrm{H}), 0.91(\mathrm{~d}, J=6.2 \mathrm{~Hz}, 3 \mathrm{H}) ;{ }^{13} \mathrm{C} \mathrm{NMR}_{\left(\mathrm{CDCl}_{3}\right) \delta}$ 176.1, 152.6, 135.5, 133.2, 132.9, 132.8, 129.7, 128.6, 127.7, 125.5, 78.8, 75.1, 68.4, 55.0, 40.7, 37.5, 26.7, 19.1, 14.2, 13.2, 9.1; El-HRMS m/z 560.2828 (calcd for $\mathrm{C}_{33} \mathrm{H}_{42} \mathrm{NO}_{5} \mathrm{Si}(\mathrm{M}+\mathrm{H})^{+} 560.2832$ ); Anal. Calcd for $\mathrm{C}_{33} \mathrm{H}_{41} \mathrm{NO}_{5} \mathrm{Si}$ : C, 
70.81; $\mathrm{H}, 7.38 ; \mathrm{N}, 2.50$. Found: $\mathrm{C}, 70.54 ; \mathrm{H}, 7.35 ; \mathrm{N}, 2.45$. A sample suitable for $\mathrm{X}$-ray diffraction analysis[10]was obtained by recrystallization from hot methanol.

\subsection{Methyl (2R,3S,4S)-5-t-butyldiphenylsilyloxy-3-hydroxy-2,4-dimethylpentanoate ((+)-}

9)

To a freshly prepared solution of methanolic sodium methoxide $(1.9 \mathrm{~g} \mathrm{Na} / 350 \mathrm{~mL} \mathrm{MeOH})$ at $0^{\circ} \mathrm{C}$ was slowly added a solution of (+)-8 (45.8 g, $81.8 \mathrm{mmol})$ in THF $(250 \mathrm{~mL})$. When 8 was no longer observed by TLC monitoring (ca. $15 \mathrm{~min}$ ), saturated aqueous $\mathrm{NH}_{4} \mathrm{Cl}$ was added and the heterogenous mixture was partitioned between $\mathrm{CH}_{2} \mathrm{Cl}_{2} / \mathrm{H}_{2} \mathrm{O}$. The layers were separated and the aqueous layer was extracted with $\mathrm{CH}_{2} \mathrm{Cl}_{2}$. All of the $\mathrm{CH}_{2} \mathrm{Cl}_{2}$ phases were combined, dried $\left(\mathrm{MgSO}_{4}\right)$ and concentrated. The residue was purified by flash chromatography (hexanes:ethyl acetate $=10: 1$ ) to give (+)-9 as a viscous oil (26.4 g, 78\%): $[\alpha]_{\mathrm{D}}+6.8$ (c 1.0, $\mathrm{CHCl}_{3}$ ); $\mathrm{R}_{f} 0.5$ (hexanes:ethyl acetate $\left.=4: 1\right) ;{ }^{1} \mathrm{H}$ NMR $\left(\mathrm{CDCl}_{3} / \mathrm{D}_{2} \mathrm{O}\right) \delta 7.69-7.65(\mathrm{~m}, 4 \mathrm{H}), 7.44-7.36(\mathrm{~m}, 6 \mathrm{H}), 3.96(\mathrm{dd}, \mathrm{J}=3.7$, $8.4 \mathrm{~Hz}, 1 \mathrm{H}), 3.83(\mathrm{~d}, J=4.2,10.3 \mathrm{~Hz}, 1 \mathrm{H}), 3.70(\mathrm{~d}, J=6.3,10.3 \mathrm{~Hz}, 1 \mathrm{H}), 3.70(\mathrm{~s}, 3 \mathrm{H}), 2.63(\mathrm{dq}, J=7.0,3.7 \mathrm{~Hz}, 1 \mathrm{H})$, $1.83-1.73(\mathrm{~m}, 1 \mathrm{H}), 1.21(\mathrm{~d}, \mathrm{~J}=7.0 \mathrm{~Hz}, 3 \mathrm{H}), 1.05(\mathrm{~s}, 9 \mathrm{H}), 0.86(\mathrm{~d}, \mathrm{~J}=7.0 \mathrm{~Hz}, 3 \mathrm{H}) ; \mathrm{MS}\left(\mathrm{Cl} / \mathrm{NH}_{3}\right) \mathrm{m} / \mathrm{z} 415(\mathrm{M}+\mathrm{H})^{+}, 432$ $\left(\mathrm{M}+\mathrm{NH}_{4}\right)^{+}$.

\subsection{Methyl (2R,3S,4S)-5-t-butyldiphenylsilyloxy-3-[methoxy(methoxy)]-2,4- dimethylpentanoate $((+)-10)$}

To a solution of (+)-9 $(26.1 \mathrm{~g}, 62.9 \mathrm{mmol})$ in freshly distilled diisopropylethylamine $(125 \mathrm{~mL})$ at $0^{\circ} \mathrm{C}$ was slowly added, via a syringe, chloromethyl methyl ether $(24.0 \mathrm{~mL}, 315 \mathrm{mmol})$. The reaction mixture was warmed to rt, and then heated at $38^{\circ} \mathrm{C}$ for $18 \mathrm{~h}$. After cooling to rt, the reaction mixture was diluted with $\mathrm{CH}_{2} \mathrm{Cl}_{2}$ and washed with $0.25 \mathrm{~N} \mathrm{HCl}(2 \times 160 \mathrm{~mL})$, followed by saturated aqueous $\mathrm{NaHCO}_{3}$, and brine: $\mathrm{H}_{2} \mathrm{O}(1: 1)$, dried $\left(\mathrm{MgSO}_{4}\right)$ and concentrated. The residue was purified by flash chromatography (hexanes:ethyl acetate $=10: 1)$ to give $(+)-10$ as a colorless oil which solidified upon storing in the freezer (27.5 g, 95\%): $\mathrm{mp} 54.5-55.5^{\circ} \mathrm{C} ;[\alpha]_{\mathrm{D}}+4\left(\mathrm{c} 0.9, \mathrm{CHCl}_{3}\right)$; $\mathrm{R}_{f} 0.60$ (hexanes:ethyl acetate $\left.=4: 1\right) ;{ }^{1} \mathrm{H}$ NMR $\left(\mathrm{CDCl}_{3}\right) \delta 7.67-7.64(\mathrm{~m}, 4 \mathrm{H}), 7.44-7.34(\mathrm{~m}, 6 \mathrm{H}), 4.53(\mathrm{~d}, \mathrm{~J}=6.6 \mathrm{~Hz}$, $1 \mathrm{H}), 4.45(\mathrm{~d}, J=6.6 \mathrm{~Hz}, 1 \mathrm{H}), 3.93(\mathrm{dd}, J=3.7,7.8 \mathrm{~Hz}, 1 \mathrm{H}), 3.73(\mathrm{~d}, J=4.2,10.3 \mathrm{~Hz}, 1 \mathrm{H}), 3.66(\mathrm{~s}, 3 \mathrm{H}), 3.61(\mathrm{dd}, J=6.0$, $10.0 \mathrm{~Hz}, 1 \mathrm{H}), 3.19(\mathrm{~s}, 3 \mathrm{H}), 2.70(\mathrm{dq}, J=7.0,3.7 \mathrm{~Hz}, 1 \mathrm{H}), 1.90-1.80(\mathrm{~m}, 1 \mathrm{H}), 1.14(\mathrm{~d}, J=7.0 \mathrm{~Hz}, 3 \mathrm{H}), 1.07(\mathrm{~s}, 9 \mathrm{H}), 0.99$ (d, J=7.0 Hz, 3H); MS (FAB) m/z $497(\mathrm{M}+\mathrm{K})^{+}$; Anal. Calcd for $\mathrm{C}_{26} \mathrm{H}_{38} \mathrm{O}_{5} \mathrm{Si}$ : C, 68.08; $\mathrm{H}, 8.35$. Found: $\mathrm{C}, 67.93 ; \mathrm{H}, 8.24$.

\section{8. (2S,3R,4S)-5-t-Butyldiphenylsilyloxy-3[(methoxy)methoxy]-2,4-dimethyl-1-pentanol} $((+)-11)$

To a solution of $(+)-10(26.7 \mathrm{~g}, 58.3 \mathrm{mmol})$ in THF $(120 \mathrm{~mL})$ at $-78^{\circ} \mathrm{C}$ was added dropwise a solution of DIBAL (128 mL, $1.0 \mathrm{M}$ in toluene, $128 \mathrm{mmol}$ ). The mixture was stirred for $15 \mathrm{~min}$, and then methanol was cautiously added until gas evolution ceased. The mixture was warmed to rt and partitioned between ethyl acetate and $1 \mathrm{M}$ potassium sodium tartrate. The separated organic layer was washed with fresh $1 \mathrm{M}$ potassium sodium tartrate, and the combined aqueous phases were extracted with $\mathrm{CH}_{2} \mathrm{Cl}_{2}$. All of the organic phases were combined, dried $\left(\mathrm{MgSO}_{4}\right)$, and concentrated. The residue was purified by flash chromatography (hexanes:ethyl acetate=8:1 to 4:1 gradient) to give (+)-11 as a viscous oil (23.0 g, 92\%): $[\alpha]_{D}+54$ (c 1.0, $\mathrm{CHCl}_{3}$ ); $\mathrm{R}_{f} 0.25$ (hexanes:ethyl acetate=4:1); ${ }^{1} \mathrm{H}$ NMR $\left(\mathrm{CDCl}_{3} / \mathrm{D}_{2} \mathrm{O}\right)$ 8 7.66-7.62 (m, 4H), 7.45-7.34 (m, 6H), $4.68(\mathrm{~d}, \mathrm{~J}=6.6 \mathrm{~Hz}, 1 \mathrm{H}), 4.43(\mathrm{~d}, \mathrm{~J}=6.6$ $\mathrm{Hz}, 1 \mathrm{H}), 3.75-3.67(\mathrm{~m}, 3 \mathrm{H}), 3.61(\mathrm{~d}, J=7.7 \mathrm{~Hz}, 1 \mathrm{H}), 3.34(\mathrm{~s}, 3 \mathrm{H}), 1.96-1.87(\mathrm{~m}, 1 \mathrm{H}), 1.87-1.77(\mathrm{~m}, 1 \mathrm{H}), 1.08(\mathrm{~s}, 9 \mathrm{H})$, $0.94\left(\mathrm{~d}, J=7.0 \mathrm{~Hz}, 3 \mathrm{H}\right.$ ), 0.76 (d, J=7.0 Hz, 3H); HRMS (Cl) m/z 431.2603 (calcd for $\mathrm{C}_{25} \mathrm{H}_{39} \mathrm{O}_{4} \mathrm{Si}(\mathrm{M}+\mathrm{H})^{+} 431.2618$; Anal. Calcd for $\mathrm{C}_{25} \mathrm{H}_{38} \mathrm{O}_{4} \mathrm{Si}$ : C, 69.26; $\mathrm{H}, 8.90$. Found: $\mathrm{C}, 69.72 ; \mathrm{H}, 8.89$. 
2.9. (2S,3R,4S)-1-[(Benzyloxy)methoxy]-5-t-butyldiphenylsilyloxy-3[(methoxy)methoxy]-

2,4-dimethyl-1-pentane $((+)-12)$

To a solution of $(+)-11(3.34 \mathrm{~g}, 7.76 \mathrm{mmol})$ in diisopropylamine $(31 \mathrm{~mL})$ at $0^{\circ} \mathrm{C}$ was added dropwise benzyloxymethyl chloride $(8.0 \mathrm{~mL}, 46.6 \mathrm{mmol})$. The reaction mixture was gradually warmed to rt and stirred for $18 \mathrm{~h}$. The mixture was then heated at a gentle reflux for $24 \mathrm{~h}$. The mixture was cooled and $2 \mathrm{~N}$ aqueous $\mathrm{HCl}$ was carefully added. The mixture was extracted with ethyl acetate and the combined extracts washed with $2 \mathrm{~N}$ aqueous $\mathrm{HCl}$, brine, dried $\left(\mathrm{MgSO}_{4}\right)$, and concentrated. The residue was purified by flash chromatography (hexanes:ethyl acetate $=20: 1$ ) to give (+)-12 as an oil $\left(2.95 \mathrm{~g}, 69 \%\right.$ ): $[\alpha]_{\mathrm{D}}+3.5$ (c 1.4, $\left.\mathrm{CHCl}_{3}\right) ; \mathrm{R}_{f} 0.17$ (hexanes:ethyl acetate=15:1); ${ }^{1} \mathrm{H} \mathrm{NMR}\left(\mathrm{CDCl}_{3}\right)$ 8 7.67-7.63 (m, 4H), 7.43-7.27 (m, 11H), 4.97-4.49 (m, 6H), 3.74 (dd, J=3.9, 9.7 $\mathrm{Hz}, 1 \mathrm{H}), 3.64-3.44(\mathrm{~m}, 4 \mathrm{H}), 3.20(\mathrm{~s}, 3 \mathrm{H}), 2.30-1.84(\mathrm{~m}, 2 \mathrm{H}), 1.07(\mathrm{~s}, 9 \mathrm{H}), 0.98(\mathrm{~d}, J=7.0 \mathrm{~Hz}, 3 \mathrm{H}), 0.88(\mathrm{~d}, J=7.0 \mathrm{~Hz}$, $3 \mathrm{H}) ; \mathrm{MS}\left(\mathrm{Cl} / \mathrm{NH}_{3}\right) \mathrm{m} / \mathrm{z} 568\left(\mathrm{M}+\mathrm{NH}_{4}\right)^{+}$; Anal. Calcd for $\mathrm{C}_{33} \mathrm{H}_{46} \mathrm{O}_{5} \mathrm{Si}: \mathrm{C}, 71.96 ; \mathrm{H}, 8.42$. Found: $\mathrm{C}, 71.83 ; \mathrm{H}, 8.26$.

\subsection{0. (2S,3S,4S)-5-[(Benzyloxy)methoxy]-3-[(methoxy)methoxy]-2,4-dimethyl-1-pentanol} $((-)-13)$

To a solution of $(+)-12(2.50 \mathrm{~g}, 4.65 \mathrm{mmol})$ in THF $(18.5 \mathrm{~mL})$ at $0^{\circ} \mathrm{C}$ was added a solution of tetrabutylammonium fluoride in THF (1.0 M, $4.9 \mathrm{~mL}, 4.9 \mathrm{mmol})$. Thr reaction mixture was warmed to rt and stirred for $3 \mathrm{~h}$. The reaction mixture was poured into water and extracted with ether, the combined extracts dried $\left(\mathrm{MgSO}_{4}\right)$, and concentrated. The residue was purified by flash chromatography (hexanes:ethyl acetate=4:1) to give (-)-13 as an oil (1.21 g, 83\%): $[\alpha]_{D}-42$ (c 1.2, $\left.\mathrm{CHCl}_{3}\right) ; \mathrm{R}_{f} 0.33$ (hexanes:ethyl acetate=2:1); ${ }^{1} \mathrm{H} \mathrm{NMR}\left(\mathrm{CDCl}_{3}\right) \delta 7.36-7.27(\mathrm{~m}, 5 \mathrm{H})$, 4.79-4.61 (m, 6H), 3.88-3.81 (m, 1H), 3.65 (dd, J=2.2, 9.2 Hz, 1H), 3.56-3.47 (m, 3H), $3.43(\mathrm{~s}, 3 \mathrm{H}), 2.93(\mathrm{t}, \mathrm{J}=6.8$ $\mathrm{Hz}, \mathrm{OH}), 2.07-1.97(\mathrm{~m}, 1 \mathrm{H}), 1.88-1.79(\mathrm{~m}, 1 \mathrm{H}), 0.96(\mathrm{~d}, \mathrm{~J}=7.0 \mathrm{~Hz}, 3 \mathrm{H}), 0.88(\mathrm{~d}, \mathrm{~J}=7.0 \mathrm{~Hz}, 3 \mathrm{H}) ; \mathrm{MS}\left(\mathrm{Cl} / \mathrm{NH}_{3}\right) \mathrm{m} / \mathrm{z} 330$ $\left(\mathrm{M}+\mathrm{NH}_{4}\right)^{+}$; Anal. Calcd for $\mathrm{C}_{17} \mathrm{H}_{28} \mathrm{O}_{5}: \mathrm{C}, 65.36 ; \mathrm{H}, 9.03$. Found: $\mathrm{C}, 65.17 ; \mathrm{H}, 9.08$.

\subsection{1. (2R,3R,4S)-5-[(Benzyloxy)methoxy]-3-[(methoxy)methoxy]-2,4-dimethylpentanal} $((-)-14)$

To a solution of oxalyl chloride $(1.4 \mathrm{~mL}, 16.5 \mathrm{mmol})$ in $\mathrm{CH}_{2} \mathrm{Cl}_{2}(25 \mathrm{~mL})$ cooled to $-78^{\circ} \mathrm{C}$ was added DMSO $(2.1 \mathrm{~mL}$, $30 \mathrm{mmol})$, followed by dropwise addition of a solution of (-)-13 (4.7 g, $15 \mathrm{mmol})$ in $\mathrm{CH}_{2} \mathrm{Cl}_{2}(30 \mathrm{~mL})$. The reaction mixture was stirred for $15 \mathrm{~min}$ and then triethylamine $(6.3 \mathrm{~mL}, 45 \mathrm{mmol})$ was added. The reaction mixture was stirred at $-78^{\circ} \mathrm{C}$ for $15 \mathrm{~min}$, warmed to rt, and a solution of $1 \mathrm{~N} \mathrm{HCl}$ was added. The phases were separated, and the organic phase washed with saturated aqueous $\mathrm{NaHCO}_{3}$, brine, dried $\left(\mathrm{MgSO}_{4}\right)$, and concentrated. The residue was purified by flash chromatography (hexanes:ethyl acetate $=8: 1$ ) to give $(-)-14$ as an oil $(3.93 \mathrm{~g}, 84 \%):[\alpha]_{D}-27$ (c 0.9, $\left.\mathrm{CHCl}_{3}\right) ; \mathrm{R}_{f} 0.17$ (hexanes:ethyl acetate=8:1); ${ }^{1} \mathrm{H} \mathrm{NMR}\left(\mathrm{CDCl}_{3}\right) \delta 9.72(\mathrm{~d}, J=2.9 \mathrm{~Hz}, 1 \mathrm{H}), 7.42-7.26(\mathrm{~m}, 5 \mathrm{H})$, $4.75(\mathrm{~s}, 2 \mathrm{H}), 4.69(\mathrm{~d}, J=6.8 \mathrm{~Hz}, 1 \mathrm{H}), 4.60(\mathrm{~d}, J=6.8 \mathrm{~Hz}, 1 \mathrm{H}), 4.60(\mathrm{~m}, 2 \mathrm{H}), 3.94(\mathrm{dd}, J=3.3,7.4 \mathrm{~Hz}, 1 \mathrm{H}), 3.50$ (d, J=7.0 $\mathrm{Hz}, 2 \mathrm{H}), 3.33(\mathrm{~s}, 3 \mathrm{H}), 2.70-2.60(\mathrm{~m}, 1 \mathrm{H}), 2.04-1.97(\mathrm{~m}, 1 \mathrm{H}), 1.07(\mathrm{~d}, \mathrm{~J}=7.0 \mathrm{~Hz}, 3 \mathrm{H}), 0.94(\mathrm{~d}, \mathrm{~J}=7.0 \mathrm{~Hz}, 3 \mathrm{H}) ; \mathrm{MS}$ $\left(\mathrm{Cl} / \mathrm{NH}_{3}\right) \mathrm{m} / \mathrm{z} 328\left(\mathrm{M}+\mathrm{NH}_{4}\right)^{+}$.

\subsection{Ethyl (2E,4S,5S,6S)-7-[(benzyloxy)methoxy]-5-[(methoxy)methoxy]-4,6-dimethyl-2- heptenoate (15)}

To a slurry of $\mathrm{NaH}$ (415 mg, $13.8 \mathrm{mmol}, 80 \%$ dispersion in mineral oil) in THF $(25 \mathrm{~mL})$ was added dropwise triethyl phosphonoacetate $(2.7 \mathrm{~mL}, 13.8 \mathrm{mmol})$. After $15 \mathrm{~min}$, a solution of (-)-14 (3.9 g, $12.6 \mathrm{mmol})$ in THF (25 $\mathrm{mL}$ ) was added. The reaction mixture was stirred for $18 \mathrm{~h}$ and then carefully quenched with $1 \mathrm{~N}$ aqueous $\mathrm{HCl}$ $(140 \mathrm{~mL})$. The phases were separated, and the aqueous phase extracted with ether. The combined organic phases were dried $\left(\mathrm{MgSO}_{4}\right)$ and concentrated. The residue was purified by flash chromatography (hexanes:ethyl acetate $=12: 1$ ) to give 15 as an oil (4.2 g, 88\%): $R_{f} 0.42$ (hexanes:ethyl acetate $\left.=4: 1\right) ;{ }^{1} \mathrm{H}$ NMR $\left(\mathrm{CDCl}_{3}\right) \delta 7.40-7.26$ $(\mathrm{m}, 5 \mathrm{H}), 7.03(\mathrm{dd}, \mathrm{J}=8.5,15.5 \mathrm{~Hz}, 1 \mathrm{H}), 5.85(\mathrm{~d}, J=15.5 \mathrm{~Hz}, 1 \mathrm{H}), 4.75(\mathrm{~s}, 2 \mathrm{H}), 4.64(\mathrm{~d}, \mathrm{~J}=6.7 \mathrm{~Hz}, 1 \mathrm{H}), 4.60(\mathrm{~s}, 2 \mathrm{H}), 4.58$ 
(d, J=6.7 Hz, 1H), $4.18(\mathrm{q}, J=7.0 \mathrm{~Hz}, 2 \mathrm{H}), 3.60-3.46(\mathrm{~m}, 3 \mathrm{H}), 3.36(\mathrm{~s}, 3 \mathrm{H}), 2.66-2.53(\mathrm{~m}, 1 \mathrm{H}), 2.20-1.92(\mathrm{~m}, 1 \mathrm{H})$, $1.28(\mathrm{t}, J=7.0 \mathrm{~Hz}, 3 \mathrm{H}), 1.07(\mathrm{~s}, 3 \mathrm{H}), 0.94(\mathrm{~d}, \mathrm{~J}=7.0 \mathrm{~Hz}, 3 \mathrm{H}) ; \mathrm{MS}\left(\mathrm{Cl} / \mathrm{NH}_{3}\right) \mathrm{m} / z 398\left(\mathrm{M}+\mathrm{NH}_{4}\right)^{+}$.

\subsection{Ethyl (4S,5S,6S)-7-[(benzyloxy)methoxy]-5-[(methoxy)methoxy]-4,6-} dimethylheptanoate ((-)-16)

A solution of $15(4.2 \mathrm{~g}, 11 \mathrm{mmol})$ and $10 \% \mathrm{Pd} / \mathrm{C}(360 \mathrm{mg})$ in ethyl acetate $(22 \mathrm{~mL})$ was stirred under an atmosphere of $\mathrm{H}_{2}(1 \mathrm{~atm})$ for $14 \mathrm{~h}$. The reaction mixture was filtered and concentrated. The residue was purified by flash chromatography (hexanes:ethyl acetate=15:1) to give 16 as an oil $\left(3.3 \mathrm{~g}, 79 \%\right.$ ): $[\alpha]_{\mathrm{D}}-9.2$ (c 1.0, $\mathrm{CHCl}_{3}$ ); $\mathrm{R}_{f} 0.42$ (hexanes:ethyl acetate=4:1); ${ }^{1} \mathrm{H} \mathrm{NMR}\left(\mathrm{CDCl}_{3}\right) \delta 7.38-7.26(\mathrm{~m}, 5 \mathrm{H}), 4.76(\mathrm{~s}, 2 \mathrm{H}), 4.68(\mathrm{~d}, \mathrm{~J}=6.6 \mathrm{~Hz}, 1 \mathrm{H}), 4.63$ (d, J=6.6 Hz, 1H), $4.60(\mathrm{~s}, 2 \mathrm{H}), 4.12(\mathrm{q}, J=7.0 \mathrm{~Hz}, 2 \mathrm{H}), 3.51-3.38(\mathrm{~m}, 3 \mathrm{H}), 3.39(\mathrm{~s}, 3 \mathrm{H}), 2.41$ (ddd, J=5.5, 10.3, 15.6 $\mathrm{Hz}, 1 \mathrm{H}), 2.26$ (ddd, J=6.4, 9.7, $15.6 \mathrm{~Hz}, 1 \mathrm{H}), 2.04-1.89(\mathrm{~m}, 2 \mathrm{H}), 1.76-1.64(\mathrm{~m}, 1 \mathrm{H}), 1.51-1.39(\mathrm{~m}, \mathrm{H}), 1.25(\mathrm{t}, J=7.0$ $\mathrm{Hz}, 3 \mathrm{H}), 0.92(\mathrm{~d}, J=7.0 \mathrm{~Hz}, 3 \mathrm{H}), 0.90(\mathrm{~d}, \mathrm{~J}=7.0 \mathrm{~Hz}, 3 \mathrm{H}) ;{ }^{13} \mathrm{C} \mathrm{NMR}\left(\mathrm{CDCl}_{3}\right) \delta 173.9,137.9,128.4,127.8,127.6,98.4$, $94.8,83.4,71.1,69.4,60.2,56.0,35.4,35.2,32.2,27.8,16.0,14.2,11.3 ; \mathrm{MS}\left(\mathrm{Cl} / \mathrm{NH}_{3}\right) \mathrm{m} / z 400\left(\mathrm{M}+\mathrm{NH}_{4}\right)^{+}$; ElHRMS $m / z 321.2057$ (calcd for $\left.\mathrm{C}_{19} \mathrm{H}_{29} \mathrm{O}_{4}\left(\mathrm{M}-\mathrm{OCH}_{2} \mathrm{OCH}_{3}\right)^{+} 321.2066\right)$.

\subsection{4. (4S,5S,6S)-7-[(Benzyloxy)methoxy]-5-[(methoxy)methoxy]-4,6-dimethylheptanoic acid (17)}

A solution of $\mathrm{KOH}(245 \mathrm{mg}, 4.3 \mathrm{mmol})$ in water $(3 \mathrm{~mL})$ and ethanol $(9 \mathrm{~mL})$ was added to $16(1.38 \mathrm{~g}, 3.6 \mathrm{mmol})$. The reaction mixture was stirred for $5 \mathrm{~h}$. The mixture was acidified with excess $2 \mathrm{~N} \mathrm{HCl}$ and extracted with $\mathrm{CH}_{2} \mathrm{Cl}_{2}$. All of the organic phases were combined, dried $\left(\mathrm{MgSO}_{4}\right)$, and concentrated. The residue was purified by flash chromatography $\left(\mathrm{CHCl}_{3}: \mathrm{MeOH}=98: 2\right)$ to give 17 as an oil $(650 \mathrm{mg}, 51 \%):{ }^{1} \mathrm{H} \mathrm{NMR}\left(\mathrm{CDCl}_{3}\right) \delta 7.36-7.26(\mathrm{~m}$, $5 \mathrm{H}), 4.76(\mathrm{~s}, 2 \mathrm{H}), 4.68(\mathrm{~d}, J=6.6 \mathrm{~Hz}, 1 \mathrm{H}), 4.63(\mathrm{~d}, \mathrm{~J}=6.6 \mathrm{~Hz}, 1 \mathrm{H}), 4.61(\mathrm{~s}, 2 \mathrm{H}), 3.52-3.38(\mathrm{~m}, 3 \mathrm{H}), 3.39(\mathrm{~s}, 3 \mathrm{H}), 2.45$ (ddd, J=5.5, 10.3, 16.0 Hz, 1H), 2.36 (ddd, J=6.9, 9.9, $16.0 \mathrm{~Hz}, 1 \mathrm{H}), 2.05-1.91(\mathrm{~m}, 2 \mathrm{H}), 1.78-1.68(\mathrm{~m}, 1 \mathrm{H}), 1.52-$ $1.41(\mathrm{~m}, \mathrm{H}), 0.92(\mathrm{~d}, \mathrm{~J}=6.7 \mathrm{~Hz}, 3 \mathrm{H}), 0.91(\mathrm{~d}, \mathrm{~J}=6.6 \mathrm{~Hz}, 3 \mathrm{H}) ;{ }^{13} \mathrm{C} \mathrm{NMR}\left(\mathrm{CDCl}_{3}\right) \delta 179.7,137.8,128.4,127.8,127.6$, 98.3, 94.8, 83.4, 71.0, 69.4, 65.8, 56.0, 35.2, 31.8, 27.5, 15.9, 15.2; $\mathrm{MS}\left(\mathrm{Cl} / \mathrm{NH}_{3}\right) \mathrm{m} / z 372\left(\mathrm{M}+\mathrm{NH}_{4}\right)^{+}$; Anal. Calcd for $\mathrm{C}_{19} \mathrm{H}_{30} \mathrm{O}_{6}: \mathrm{C}, 64.38 ; \mathrm{H}, 8.53$. Found: $\mathrm{C}, 63.90 ; \mathrm{H}, 8.49$.

\section{Acknowledgements}

Financial support for this work was provided by the National Institutes of Health (GM-42641). The high resolution mass-spectral determination for (-)-16 was made at the Washington University Resource for Mass Spectrometry. The authors thank Mr Rodger Henry for obtaining the X-ray crystal structure of (+)-8 and $\mathrm{Mr}$ Bireshwar Dasgupta for obtaining the ${ }^{13} \mathrm{C}$ NMR spectra of 8, 16, and 17.

\section{References}

1 (a) Hayashi, M.; Ohara, H.; Ohno, M.; Sakakibara, H.; Satoi, S.; Harada, K.; Suzuki, M. J. Antibiotics 1981, 34, 1075-7; (b) Hayashi, M.; Kinoshita, K.; Satoi, S.; Nakatsu, K. J. Antibiotics 1982, 35, 1243-4; (c) Suzuki, H.; Takenaka, S.; Kinoshita, K.; Morohoshi, T. J. Antibiotics 1990, 43, 1508-11; (d) Kinoshita, K.; Takenaka, S.; Hayashi, M. J. Chem. Soc., Chem. Commun. 1988, 943-5.

$\underline{2}$ (a) Woo, P. W. K.; Rubin, J. R. Tetrahedron 1996, 52, 3857-72; (b) Ganguly, A. K.; Liu, Y. T.; Sarre, O.; Jaret, R. S.; McPhail, A. T.; Onan, K. K. Tetrahedron Lett. 1980, 21, 4699-4702; (c) Omura, S.; Matsubara, H.; Nakagawa, A.; Furusaki, A.; Matsumoto, T. J. Antibiotics 1980, 33, 915-7.

$\underline{3}$ (a) Honda, M.; Katsuki, T.; Yamaguchi, M. Tetrahedron Lett. 1984, 25, 3857-60; (b) Suzuki, K.; Tomooka, K.; Katayama, E.; Matsumoto, T.; Tsuchihashi, G. J. Am. Chem. Soc. 1986, 108, 5221-29.

$\underline{4}$ (a) Takano, S.; Sekiguchi, Y.; Shimazaki, Y.; Ogasawara, K. Tetrahedron Lett. 1989, 30, 4001-2; (b) Takano, S.; Sekiguchi, Y.; Shimazaki, Y.; Ogasawara, K. Heterocycles 1992, 33, 713-42.

$\underline{5}$ Wasicak, J. T.; Craig, R. A.; Henry, R.; Dasgupta, B.; Li, H.; Donaldson, W. A. Tetrahedron1997, 53, 4185-98. 
$\underline{6}$ Hoffmann, R. W. Angew. Chem., Int. Ed. Engl. 1987, 26, 489.

$\underline{7}$ (a) Ley, S. V.; Anthony, N. J.; Armstrong, A.; Brasca, M. G.; Clarke, T.; Culshaw, D.; Greck, C.; Brice, P.; Jones, A. B.; Lygo, B.; Madin, A.; Sheppard, R. N.; Slawin, A. M. Z.; Williams, D. J. Tetrahedron 1989, 45, 7161-94; (a) Heathcock, C. H.; Young, S. D.; Hagen, J. P.; Pilli, R.; Badertscher, U. J. Org. Chem. 1985, 50, 20952105; (b) Roush, W. R.; Palkowitz, A. D.; Ando, K. J. Am. Chem. Soc. 1990, 112, 6348-59.

8 Evans, D. A.; Dow, R. L.; Shih, T. L.; Takacs, J. M.; Zahler, R. J. Am. Chem. Soc. 1990, 112, 5290-5313.

9 Evans, D. A.; Nelson, J. V.; Vogel, E.; Taber, T. R. J. Am. Chem. Soc. 1981, 103, 3099-3111; Roush, W. R. J. Org. Chem. 1991, 56, 4151-7. This stereotriad has been previously prepared by aldol condensation of (S)-3benzyloxy-2-methylpropanal with $N$-propionyl-4(R)-isopropyloxazolidin-2-one: Seebach, D.; Chow, H.-F.; Jackson, R. F. W.; Lawson, K.; Sutter, M. A.; Thaisrivongs, S.; Zimmerman, J. J. Am. Chem. Soc. 1985, 107, 5292-3.

10 Crystal data for (+)-8: $\mathrm{MW}=559.8, \mathrm{C}_{33} \mathrm{H}_{41} \mathrm{NO}_{5} \mathrm{Si}$, crystal dimensions $0.700 \times 0.700 \times 0.100 \mathrm{~mm}$, monoclinic, $\mathrm{P} 2_{1}$ (\#4), $a=13.836(3), b=7.2965(6), c=16.358(3) \AA, \quad b=108.14(2)^{\circ}, V=1569.5(5) \AA^{3}, Z=2, D_{\text {calc }}=1.184 \mathrm{~g} \mathrm{~cm}^{-3}$. Crystallographic data were collected with a Rigaku AFC5R diffractometer using $\mathrm{Cu}$ K $\alpha$ radiation $(\lambda=1.54178 \AA$ ). Refinement of the structure using full matrix least squares refinement of 360 parameters on 2363 reflections with $>3.00 \sigma(I)$ gave $R=0.053, R_{w}=0.064(15)$. We thank Mr Rodger Henry, Abbott Laboratories, for performing this $\mathrm{X}$-ray diffraction analysis.

Copyright (C) 1998 Elsevier Science Ltd. All rights reserved. 